\title{
ASYMMETRIC BRAGG MIRROR DESIGN FOR ORGANIC MICROCAVITY LIGHT EMITTING DIODES
}

\author{
A. B. Djurišić, ${ }^{1}$ A. D. Rakić, ${ }^{2}$ and M. L. Majewski ${ }^{2}$ \\ ${ }^{1}$ Department of Electrical and Electronic Engineering \& Department of Physics, the University of Hong Kong, \\ Pokfulam Road, Hong Kong \\ ${ }^{2}$ School of Information Technology and Electrical Engineering, The University of Queensland, Brisbane \\ Qld4072, Australia \\ E_mail: ${ }^{1}$ dalek@eee.hku.hk and ${ }^{2}$ rakic@itee.uq.edu.au
}

\begin{abstract}
In this work, we present genetic algorithm based approach for the design of asymmetric Bragg mirrors for the microcavity organic light emitting diodes (OLEDs) applications. The phase shift of the Bragg mirror is calculated using the matrix formulation for light propagation through a thin film multilayer. The objective function to be minimized is the wavelength shift in $\mathrm{Ag} / \mathrm{Alq} / \mathrm{TPD} / \mathrm{ITO} / \mathrm{Bragg}$ mirror/glass device, where ITO is indium tin oxide, $\mathrm{Alq}_{3}$ is tris (8-hydroxyquinoline) aluminum, and TPD is N,N'-disphenyl-N,N'-bis(3methylphenyl)-1,1'-disphenyl-4,4'-diamine, which are commonly used emitting and hole transport materials. We have considered $\mathrm{TiO}_{2} / \mathrm{SiO}_{2}$ and $\mathrm{Si}_{3} \mathrm{~N}_{4} / \mathrm{SiO}_{2}$ Bragg mirrors, where thickness of each layer in the mirror is determined by minimizing the emission wavelength shift using a genetic algorithm. Simulation results show that the use of asymmetric Bragg mirrors may enable reduction of the emission wavelength shift in organic microcavity devices.
\end{abstract}

\section{INTRODUCTION}

Due to low cost, high brightness, and wide viewing angle organic light emitting diodes (OLEDs) have potential for a vast number of consumer applications, ranging from displays for handheld telecomm and infocomm devices, up to military flexible displays and large area displays or lighting equipment. In order to enhance the extraction efficiency from an OLED, use of planar microcavity structures has been attracting attention in recent years. ${ }^{1-11}$ In addition to brightness enhancement, microcavity OLEDs exhibit spectral narrowing resulting in improved colour purity. However, since most organic materials have a wide emission spectrum (FWHM is typically around $100 \mathrm{~nm}$ ), microcavity organic light emitting diodes (MOLEDs) exhibit a significant drawback, i.e. variation of the emission wavelength with the viewing angle. Blue shift of the resonant wavelength of the cavity, and thusly the emission wavelength blue shift, with the increase of the viewing angle is partly due to the change of the optical path in the materials inside the cavity, and partly due to the change of the phase shift upon reflection at the mirrors. Since significant emission wavelength shifts with viewing angle in MOLEDs have been reported $\left(580 \mathrm{~nm}\right.$ to $\sim 530 \mathrm{~nm}$ at $50^{\circ}$, and $560 \mathrm{~nm}$ to $\sim 530 \mathrm{~nm}$ at $40^{\circ}, 610 \mathrm{~nm}$ to $\sim 500 \mathrm{~nm}$ at $80^{\circ}$, and $540 \mathrm{~nm}$ to $\sim 500 \mathrm{~nm}$ at $50^{\circ}{ }^{7}$ ), it is important to analyze this phenomenon and investigate ways to reduce the wavelength dependence on the viewing angle.

Typical microcavity OLED consists of one or more organic layers between two mirrors. Top mirror is usually a low work function metal cathode, while bottom mirror can be either semitransparent metal or Bragg reflector. These two typical device structures are illustrated in Fig. 1. The device structure with two metal mirrors typically results in shorter cavity length, since the bottom mirror serves as both anode and a mirror, while use of dielectric Bragg mirror requires placing transparent indium tin oxide (ITO) anode inside the cavity. However, Bragg mirrors have one significant advantage over metal mirrors. If an asymmetric Bragg mirror is used, wavelength and angle dependence of the phase shift can be tailor-made to partly compensate the change of the optical path inside the cavity with viewing angle. 
In this work, we propose the use of genetic algorithms to design a Bragg mirror which will reduce blue shift with the viewing angle in microcavity OLEDs. The device structures we will consider consist of silver cathode, tris (8-hydroxyquinoline) aluminum $\left(\mathrm{Alq}_{3}\right)$ as an emitting layer, and $\mathrm{N}, \mathrm{N}^{\prime}$-disphenyl-N, $\mathrm{N}$ '-bis(3-methylphenyl)-1, 1'disphenyl-4,4'-diamine as a hole transport layer, ITO anode, $\mathrm{TiO}_{2} / \mathrm{SiO}_{2}$ or $\mathrm{SiO}_{2} / \mathrm{Si}_{3} \mathrm{~N}_{4}$ Bragg mirror, and a glass substrate.

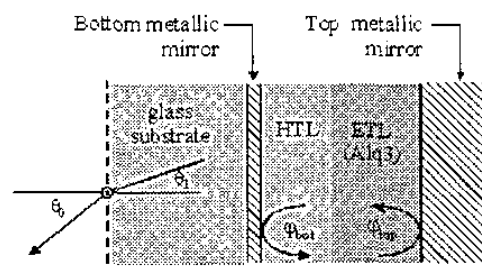

a)

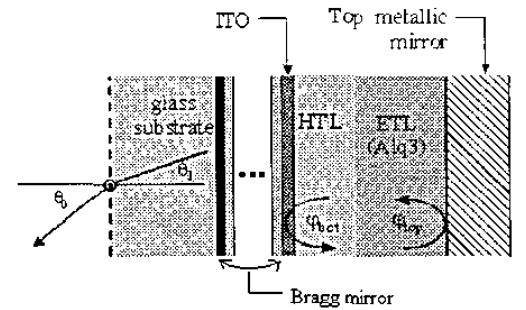

b)

Fig. 1 Two typical device structures for microcavity OLEDs a) two metal mirrors b) bottom Bragg mirror and top metal mirror.

\section{DESCRIPTION OF THE MODEL}

The resonant modes of a microcavity have to satisfy the condition that the phase change during one round trip is a multiple of $2 \pi$. In other words, for normal incidence, the following equation is valid:

$$
\sum_{i} \frac{4 \pi d_{i} n_{i}(\lambda)}{\lambda}-\varphi_{t o p}(0, \lambda)-\varphi_{b o t}(0, \lambda)=2 m \pi
$$

where $\lambda$ is the emission wavelength, $\varphi_{\text {sop }}(0, \lambda), \varphi_{\text {bor }}(0, \lambda)$ are the angle and wavelength dependent phase changes upon reflection from top and bottom mirrors, respectively, $m$ is an integer which defines the mode number, and the summation is performed over all the layers inside the cavity with thicknesses $d_{i}$ and refractive indices $n_{i}(\lambda)$. For oblique incidence, the round trip condition has the following form:

$$
\sum_{i} \frac{4 \pi d_{i} n_{i}(\lambda+\Delta \lambda) \cos \theta_{i}}{\lambda+\Delta \lambda}-\varphi_{t o p}\left(\theta_{t o p}, \lambda+\Delta \lambda\right)-\varphi_{b o t}\left(\theta_{b o t}, \lambda+\Delta \lambda\right)=2 m \pi
$$

where $\theta_{\text {top }}, \theta_{\text {bot }}$ are incidence angles at top and bottom mirrors, respectively, while $\theta_{i}$ is the angle of propagation within $i$-th organic layer, and $\varphi_{\text {top }}\left(\theta_{\text {sop }}, \lambda+\Delta \lambda\right), \varphi_{\text {bor }}\left(\left(\theta_{b o t}, \lambda+\Delta \lambda\right)\right.$ are the phase changes upon reflection from top and bottom mirrors, respectively. The angles in the equations above are related to the viewing angle outside cavity $\theta_{0}$ via Snell's law $n_{i} \sin \theta_{i}=\sin \theta_{0}$. From Eqs. (1) and (2), the following expression for the wavelength shift can be obtained:

$$
\begin{gathered}
\Delta \lambda=\frac{1}{\Delta \Phi}\left\{\sum_{i} 4 \pi d_{i}\left[n_{i}(\lambda+\Delta \lambda) \cos \theta_{i}-n_{i}(\lambda)\right]-\lambda\left(\Delta \varphi_{t o p}+\Delta \varphi_{b o t}\right)\right\}, \\
\Delta \Phi=2 m \pi+\varphi_{t o p}\left(\theta_{t o p}, \lambda+\Delta \lambda\right)+\varphi_{b o t}\left(\theta_{b o t}, \lambda+\Delta \lambda\right), \\
\Delta \varphi_{\text {top }}=\varphi_{\text {top }}\left(\theta_{t o p}, \lambda+\Delta \lambda\right)-\varphi_{\text {top }}(0, \lambda), \quad \Delta \varphi_{b o t}=\varphi_{b o t}\left(\theta_{b o t}, \lambda+\Delta \lambda\right)-\varphi_{b o t}(0, \lambda) .
\end{gathered}
$$

The wavelength shift needs.to be calculated using iterative procedurre since Eq. (3) contains several wavelength dependent terms. The starting point is the wavelength shift calculated for the case of no dispersion. The procedure converges quickly and it is terminated when the change in $\Delta \lambda$ in two consecutive iterations is smaller than $0.1 \mathrm{~nm}$. The wavelength dependence of the index of refraction for the calculation of phase change upon reflection from a metal mirror was modeled using oscillator model, with the parameters listed in Ref. 12. The 
refractive indices of $\mathrm{Alq}_{3}$ and TPD were modeled using Cauchy equation $n(\lambda)=A+B / \lambda^{2}+C / \lambda^{4}$, where the coefficients $A, B$, and $C$ were determined by fitting the data in Ref. 13. The refractive index of ITO was modeled using Lorentz-Drude model, ${ }^{14}$ while the phase change upon reflection from the Bragg mirror was calculated using matrix formalism. The refractive index of each layer was modeled using Cauchy equation, where the coefficients $A, B$, and $C$ were determined from fitting the experimental data tabulated in Ref. 15 . We have decided to perform exact calculations of the phase change upon reflection from a multilayer stack instead of using the penetration depth approximation since we will be dealing with asymmetric instead of standard $\lambda / 4$ layers and, more importantly, the penetration depth approximation is not valid in the cases when both angle and wavelength change need to be considered.

For the optimization of thickness of layers in the Bragg mirrors, we have used genetic algorithm (GA). ${ }^{16}$ Detailed procedure for Bragg mirror design using three different approaches is given in Ref. 17. In this work, we have limited our analysis to the most simple case where the materials are chosen, and only the thickness of each layer is optimized. The objective function used for minimization is given by following equation:

$$
F=\sum_{\theta_{0}} \Delta \lambda\left(\theta_{0}\right)+a\left|R_{c a l c}-R_{d}\right|
$$

where the summation is performed over viewing angles outside the cavity $\theta_{0}$ from $5^{\circ}$ to $60^{\circ}$ (with $5^{\circ}$ step), while $a$ is a constant determining the weighting of the penalty term to ensure sufficient reflectivity, $R_{\text {calc }}$ is the calculated reflectance of the Bragg mirror, and $R_{d}$ is the desired reflectance of the Bragg mirror as determined from the conditions necessary to achieve brightness enhancement as described in Ref. 17.

\section{RESULTS AND DISCUSSION}

Fig. 2 shows comparison between the emission wavelength shift in a microcavity device with two Ag mirrors, one $\mathrm{Ag}$ and one conventional $6 \mathrm{TiO}_{2} / \mathrm{SiO}_{2}$ pair Bragg mirror, and the 12 layer asymmetric mirror, which was designed using genetic algorithm. The layer thickness values (in $\mathrm{nm}$ ) are 60.2/154.6/81.2/191.5/147.0/178.0/ $165.2 / 13.4 / 25.9 / 160.1 / 83.5 / 125.9$, while the mirror reflectivity is 0.236 . The reflectivity of the asymmetric mirror is lower than that of the symmetric one, but the emission wavelength shift is significantly lower than the one for symmetric mirror. It should be pointed out that lower reflectivity does not represent a drawback, since the maximum reflectivity of the mirror for which brightness enhancement can be achieved is limited by losses and spectral width of the emitting material. ${ }^{18}$ In case of $\mathrm{Alq}_{3}$, because of its broad emission, the maximum reflectivity of the mirror should be 0.66 for $m=1$, or 0.32 for $m=2$.
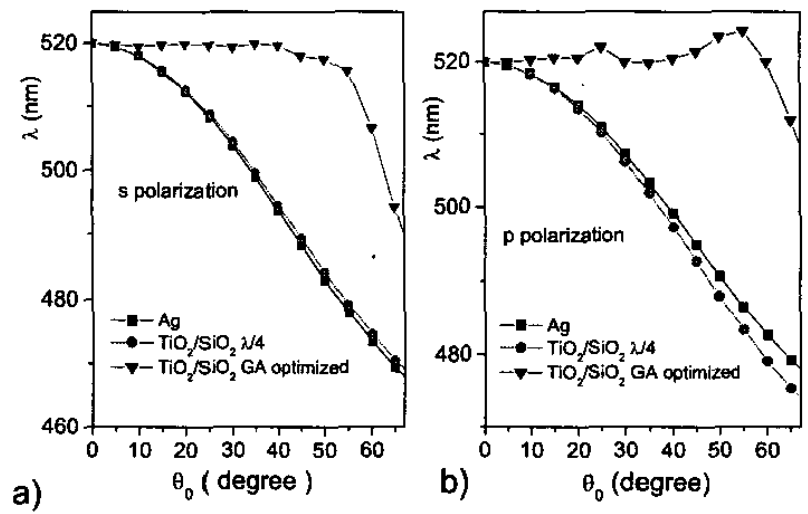

Fig.2 a) The emission wavelength shift vs. viewing angle for s polarization b) The emission wavelength shift vs. viewing angle for $\mathrm{p}$ polarization.

Fig. 3 shows comparison between the emission wavelength shifts in microcavity devices with GA optimized 8 layer $\mathrm{TiO}_{2} / \mathrm{SiO}_{2}$ and 10 layer $\mathrm{Si}_{3} \mathrm{~N}_{4} / \mathrm{SiO}_{2}$ mirrors. It can be observed that the shift compensation is slightly better for $\mathrm{TiO}_{2} / \mathrm{SiO}_{2}$ mirror, in spite of fewer layers. In addition, reflectivities of the $\mathrm{TiO}_{2} / \mathrm{SiO}_{2}$ and $\mathrm{Si}_{3} \mathrm{~N}_{4} / \mathrm{SiO}_{2}$ mirrors are $R=0.261$ and $R=0.087$, respectively. Therefore, $\mathrm{TiO}_{2} / \mathrm{SiO}_{2}$ represents a better choice for a Bragg mirror in 
MOLEDs, since fewer layers are needed to achieve acceptable reflectivity and compensation of the emission wavelength shift with the viewing angle.

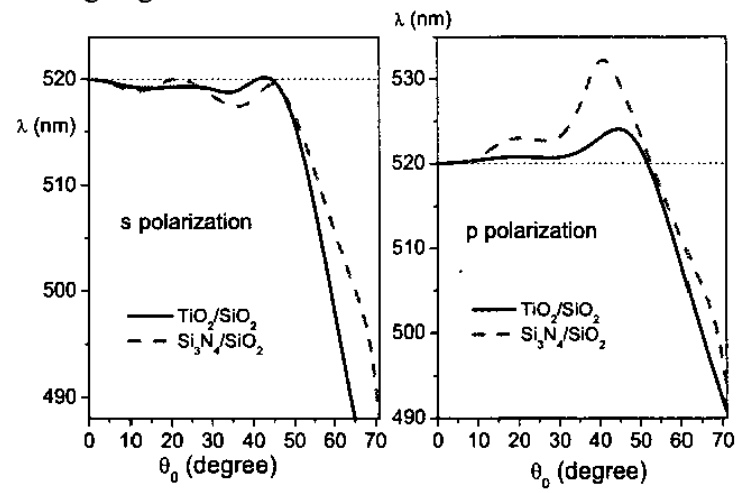

Fig.3 a) The emission wavelength shift vs. viewing angle for s polarization $b$ ) The emission wavelength shift vs. viewing angle for $\mathrm{p}$ polarization.

\section{CONCLUSION}

We presented a model for the emission wavelength dependence on the viewing angle in organic microcavity light emitting diodes. In order to minimize the emission wavelength dependence with the viewing angle, a genetic algorithm was used to design a Bragg mirror whose phase shift upon reflection can partly compensate the change of the optical path inside the cavity. The phase shift of the Bragg mirror was calculated using matrix formulation for the light propagation through a thin film multilayer. The simulation results show that the use of asymmetric Bragg mirrors is a promising method for the reduction of emission wavelength shift with the viewing angle in organic microcavity light emitting diodes.

\section{ACKNOWLEDGEMENTS}

This work has been supported by RGC Competitive Earmarked Research Grant 7056/02E.

\section{REFERENCES}

1. B. Zhang, Y. Ma, M. Xu, K. Wu, C. Huang, Y. Zhao, D. Zhuo, L. Yin, and X. Zhao, Solid State Commun. 104, 593 (1997).

2. A. Dodabalapur, L. J. Rothberg, and.T. M. Miller, Appl. Phys. Lett. 65, 2308 (1994).

3. S. Tokito, K. Noda, and Y. Taga, Appl. Phys. Lett. 68, 2633 (1996).

4. X. Y. Liu, L. X. Wang, Y. Liu, S. L. E, J. M. Zhao, D. J. Wu, Y. Q. Ning, S. L. Wu, L. J. Wang, C. J. Liang, D. X. Zhao, Z. R. Hong, D. Zhao, C. Q. Jin, X. B. Jing, F. S. Wang, W. L. Li, and S. T. Lee, Thin Solid Films 363, 204 (2000).

5. J. Grüner, F. Cacialli, and R. H. Friend, J. Appl. Phys. 80, 207 (1996).

6. R. H. Jordan, L. J. Rothberg, A. Dodabalapur, and R. E. Slusher, Appl. Phys. Lett. 69, 1997 (1996).

7. S. Dirr, S. Wiese, H.-H. Johanns, D. Ammermann, A. Böhler, W. Grahn, and W. Kowalsky, Synthetic Metals 91, 53 (1997).

8. A. Dodabalapur, L. J. Rothberg, R. H. Jordan, T. M. Miller, R. E. Slusher, and J. M. Phillips, J. Appl. Phys. 80, 6954 (1996).

9. N. Takada, T. Tsutsui, and S. Saito, Appl. Phys. Lett. 63, 2032 (1993).

10. H. Becker, S. E. Burns, N. Tessler, and R. H. Friend, J. Appl. Phys. 81, 2825 (1997).

11. N. Tessler, S. Burns, H. Becker, and R. H. Friend, Appl. Phys. Lett. 79, 556 (1997).

12. A. D. Rakić A. B. Djurišić, J.M. Elazar and M. L. Majewski, Appl. Opt. 37, 5271 (1998).

13. F. G. Celii, T. B. Horton, and D. F. Philips, J. Electron. Mater. 26, 366 (1997).

14. S. Laux, N. Kaiser, A: Zöller, R. Götzelmann, H. Lauth, and H. Bernitzki, Thin Solid Films 335

15. E. D. Palik, Ed., Handbook of Optical Constants of Solids vol.1, Academic Press, Inc., Boston, 1985.

16. A. B. Djurišić, Opt. Commun. 151, 147 (1998).

17. A. B. Djurišić, N. K. Bundaleski, and E. H. Li, Semicond. Sci. Technol. 16, 91 (2001).

18. H. Benisty, H. De Neve, and C. Weisbuch, IEEE Joumal of Quantum Electronics 34, 1612 (1998). 